Proceeding

\title{
Stereotactic radiotherapy and radiosurgery (SRT and SRS) treatment planning
}

\section{Objectives}

The objectives of Stereotactic Radiosurgery and Radiotherapy are radiation focus precisely on the tumor while minimizing radiation to nearby normal tissues. Achieving this goal requires the use of an equipment of computed tomography (CT) to purchase images to be used in treatment planning, as well as studies of magnetic resonance (MR) angiography and in some positron emission tomography images (PET). Today, these are the diagnostic equipment specially designed to be used as treatment simulators. The planning of radiotherapy or stereotactic radiosurgery requires the services of specially trainedoncologists, neurosurgeons, physical-medical and technical.

Successful treatment depends on how accurate the simulation CAT and MRI or angiography, if necessary. At this stage the patient first meet their radiation technologists, too (called "Therapists Radiation or Radiotherapy and radiology technicians"). The technician is trained to administer radiation dose prescribed by the doctor. It is also responsible for helping you achieve a comfortable position that will be the best to allow photons or electrons X-rays pass through the body part being treated. He or she will provide special accessories or foam supports for the patient to stay in the same position for each treatment. The patients also know his medical physicist. A medical physicist made the remarks on the physical aspects of the treatment plan.

Abbreviations: $\mathrm{CT}$, computed tomography; MR, magnetic resonance; PET, positron emission tomography images

\section{Being simulated}

\section{Breast patient simulation}

Some patients are confused with the notion of being simulated. Like a flight simulator allows a student pilot experience most aspects of a flight without leaving the land, or CAT radiotherapy simulator simulation, technical assistance and the radiation oncologist to design your treatment before be given some therapy. The CAT simulation allows the technical, physical, the neurosurgeon and radiation oncologist clearly see how much the patient will be treated realizing you a scan before they begin therapy (Figure 1).

\section{Patient simulation breast $\mathbf{C a}$}

During treatment simulation, technical, medical oncologist prior indication and/or neurosurgeon, puts marks on the patient's skin to aid in positioning your body and accurate source address/range $\mathrm{x}$-rays for each treatment. Often surgery technicians use markers for this purpose. It can also be tailored upon which a mask alignment reference lasers paint and also help the patient to remain still during treatment. Sometimes it can be tattooed on the skin with small black spots as a permanent mark to those who are called tattoos alignment.

\section{PET-CT in treatment planning}

PET-CT used for SRT and SRS Simulations Proper staging of tumor disease allows treatment planning to implement in cancer patients. FDG PET has been a useful diagnostic tool in the detection of
Volume 2 Issue 4 - 2015

Jasmina Alexander
Radiology and Radiation Therapy Technician, USA

Correspondence: Jasmina Alexander, Novalis Treatment Center, Panama City, Punta Pacifica John Hopkins Hospital, Basement 2, Panama, USA, Tel 507396 3162, Email jasminaalexander@radiocirugiapanama.com

Received: May 08, 2014 | Published: May 19, 2015

malignant lesions than CT or MRI in many tumor types (Figure 2). The integration of the metabolic information from PET with the anatomic data from $\mathrm{CT}$, allows increasing the diagnostic yield in the delineation of lesions by about $20-30 \%$. The PET-CT hybrid equipment represent a significant impact in the planning of radiotherapy treatments both on calculating the radiation dose to the tumor volume as a treat, as demonstrated in studies conducted with both techniques separately. FDG image delimits perfectly within the anatomic image of the tumor which areas of malignant activity and intensity of them are. This will be more effective in the treatments applied and prevent damage to healthy peritumoral tissues.

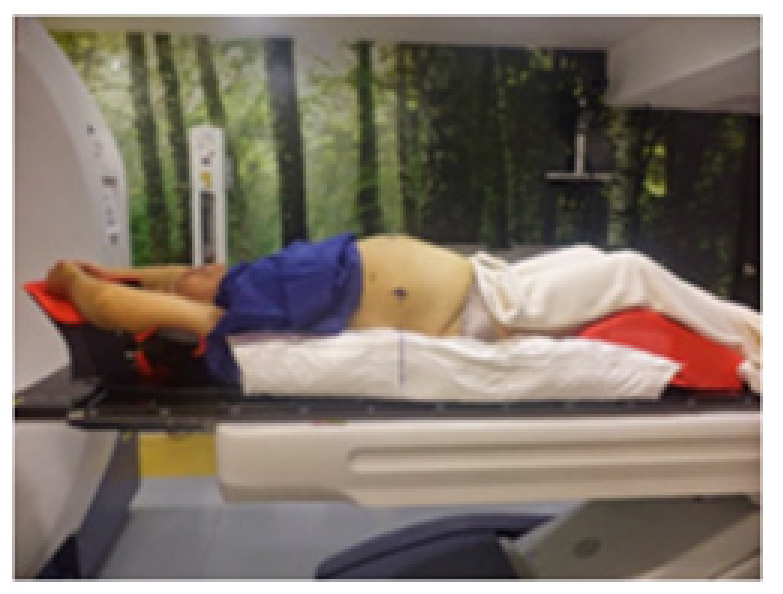

Figure I Patient simulation breast Ca.

The planning of radiotherapy based on the information provided by the PET-CT allows accurate staging of inconclusive CT injuries for introducing similar density. Moreover, it is able to detect distant lesions not previously known to PET-CT study thanks to the great sensitivity in detecting malignant disease with FDG image. 
The integration of hybrid PET-CT in the three-dimensional radiotherapy planning brings great benefits:

a. Allows more accurately define the volume when compared with CT.

b. Reduce the risk of errors in the topographic location of lesions, minimizing the dose of ionizing radiation received by non-target organs.

c. Change the current concepts of radiation therapy planning by incorporating metabolic and biological aspects of the tumor disease and not just the strictly anatomical.

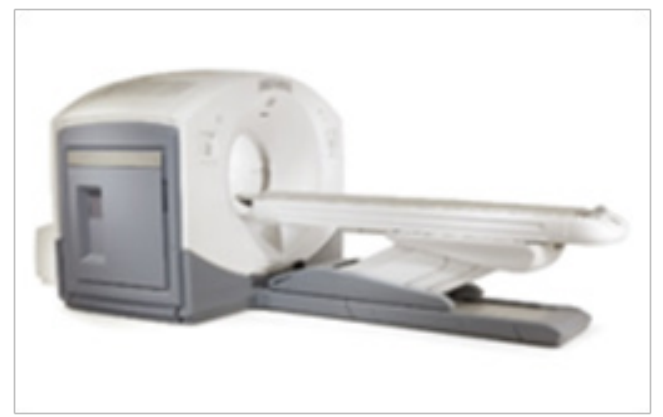

Figure 2 PET-CT used for SRT and SRS simulations.

\section{Inside the treatment room}

\section{Frameless radiosurgery}

Today, radiation oncologists often prescribed dose slightly higher than the prescribed 20 years ago, with better success in eliminating cancer x-rays. Patients are usually with fewer side effects than 20 years, largely as a result of the availability of better machines and more precise treatment planning them (Figure 3).

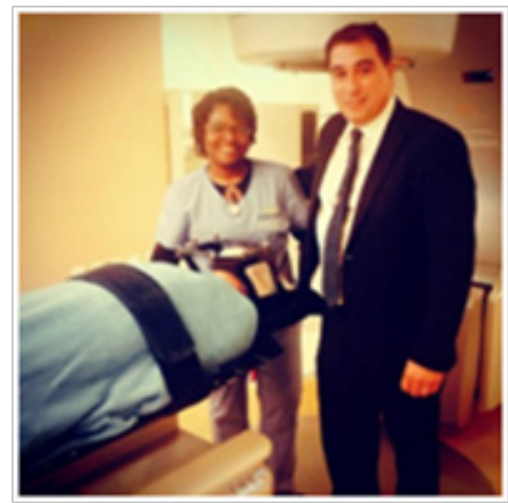

Figure 3 Frameless radio surgery.
Patients often wish to complete their therapies as quickly as possible, and occasionally wonder why it takes so many days or weeks to complete radiotherapy treatment. The duration depends mostly on the achievements of therapy. Treatments can range from a simple treatment to prevent the growth of normal tissue near a scar, for example, three weeks or more in an attempt to cure a patient with Hodgkin's disease. These are extreme cases. Radiation therapy to reduce pain in a patient with cancer that has spread to the bones, it is often administered over a period of two to three weeks. Radiation therapy given after cancer surgery to remove the few cancer cells that may have been hidden, often requires five or six weeks. During treatment, most patients continue their work and drive to the hospital every day to go to their treatments.

For personal hygiene, wash areas of your body being irradiated carefully, without allowing the water to run without rubbing marks (in case you have any marks on the skin than tattoos); for this use a soft cloth and mild soap like Dove or Ivory. Occasionally technical add more ink or marker to prevent their brands disappear during the treatment period; however, if this happens, losing care, since they are only to minimize the time it takes to position it for treatment.

Having completed their period of treatment, patients will be told by their doctor how often they should attend follow-up appointments. This will depend on the achievements of his therapy and the risk of a recurrence or side effects. Generally, most patients are tested in control appointments culminated a month after therapy and three to six months thereafter for five to ten years (Figure 4).

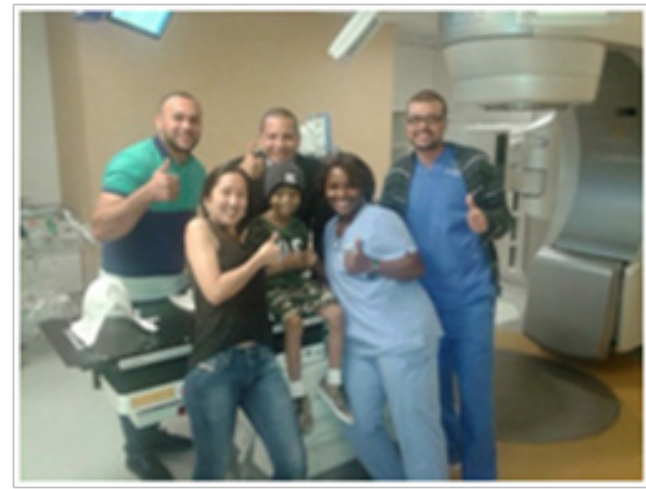

Figure 4 Having completed their period of treatment.

\section{Acknowledgments}

None.

\section{Conflicts of interest}

Author declares that there is no conflict of interest. 